\title{
Efficacy of commercial formulas in comparison with home-made formulas for enteral feeding: A critical review: Letter to the Editor
}

\author{
Georgia Tsaousi ${ }^{1}$, George Stavrou ${ }^{2,3}$, Katerina Kotzampassi*2 \\ Received: 10 Aug 2018 \\ Published: 2 Sep 2019 \\ Conflicts of Interest: None declared \\ Funding: None \\ *This work has been published under CC BY-NC-SA 1.0 license. \\ Copyright $\subseteq$ Iran University of Medical Sciences
}

Cite this article as: Tsaousi G, Stavrou G, Kotzampassi K. Efficacy of commercial formulas in comparison with home-made formulas for enteral feeding: A critical review: Letter to the Editor. Med J Islam Repub Iran. 2019 (2 Sep);33:91. https://doi.org/10.47176/mjiri.33.91

\section{Dear editor}

We have read with great interest the review article: "Efficacy of commercial formulas in comparison with homemade formulas for enteral feeding: A critical review." by Hassan-Ghomi and colleagues published in your esteemed journal in September of 2017 (1).

The authors deal with a very intriguing topic as nutrition support in hospitalized patients still remains an issue of utmost importance, though under-recognized by most healthcare systems.

Although a considerable body of evidence substantiates the beneficial role of proper nutritional support on clinical outcomes (2), there is scanty information about the comparable effect of home-made to commercial formulas for enteric feeding in different clinical settings, yet. Furthermore, the diversity of the established nutritional protocols among institutions, as well as, the use of nutritional interventions driven by health economic policies, pose extra difficulties in the extraction of a definite conclusion. The magnitude of this problem is amplified in low-income countries, in which limited financial resources eliminate treatment alternatives. This seem to be the case for the Iranian healthcare system, as authors state that no insurance cover is provided for commercial formulas and thus in Iran still home-made formulas are employed for nutritional support of hospitalized patients.

The authors made an appreciable effort to shed more light on the nutritional efficacy of either home-made enteric formulas recommended by the competent authorities or commercial ones being available in their country, by conducting a meticulous macro- and micronutrient composition analysis.
As expected commercial formulas emerged as a superior choice than home-made diets in terms of nutritional efficacy, safety and ease of preparation. Nonetheless, there is still room for further enhancement of their composition, to meet disease-oriented nutritional requirements, as in cases involving major trauma, burns or cancer. As a rule of thumb, the nutritional quality of home-made enteral feeding is graded as inferior to commercial products, attributed mainly to the high variability of nutritional composition of home-made enteral formulas. Moreover, preparation of disease-specific enteral formulas that can cover every aspect of nutritional deficits, it is a daunting if not impossible - task.

Our recently published study conducted in head and neck cancer patients receiving either commercialcomplied or standardized home-made enteral formulas comes to reinforce the aforementioned findings (3). In patients who decided to discard disease-specific commercial enteric formula - despite being advised otherwise and consumed standardized home-made feeding, a notable deterioration in their nutritional status throughout the 8month study period, occurred. Of note, the enhanced cost of commercial feeding product, in cases of no insurance cover, prevented several patients from complying with physicians' recommendations.

It should be emphasized that financial restrictions influence the quality of care in many countries - including ours - worldwide. A multidimensional approach needs to be adapted by healthcare policies to an effective management of nutritional requirements of critically ill patients. Considering that the economic burden of the prolonged hospitalization and poor clinical outcome is directly related to patients' poor nutritional status (4), it occurs that the esti-

\footnotetext{
Corresponding author: Dr Katerina Kotzampassi, kakothe@yahoo.com

1. Department of Anesthesiology and ICU, Faculty of Medicine, Aristotle University of Thessaloniki, Thessaloniki, Greece

. Department of Surgery, Faculty of Medicine, Aristotle University Thessaloniki, University Campus, Thessaloniki, Greece

3. Department of General Surgery, York Teaching Hospital, NHS Foundation Trust, York, UK
} 
mated cost-savings of a certain medical practice should be cost-effective, as well.

\section{Conflict of Interests}

The authors declare that they have no competing interests.

\section{References}

1. Hassan-Ghomi M, Nikooyeh B, Motamed S, R Neyestani T. Efficacy of commercial formulas in comparison with home-made formulas for enteral feeding: A critical review. Med J Islam Repub Iran. 2017 (5 Sep);31:55.

2. Borghi R, Dutra Araujo T, Airoldi Vieira RI, Theodoro de Souza T, Waitzberg DL. ILSI task force on enteral nutrition; estimated composition and costs of blenderized diets. Nutr Hosp. 2013;28(6):2033-2038.

3. Papakostas P, Tsaousi G, Stavrou G, Rachovitsas D, Tsiropoulos G, Rova C, et al. Percutaneous endoscopic gastrostomy feeding of locally advanced oro-pharygo-laryngeal cancer patients: Blenderized or commercial food? Oral Oncol. 2017;74:135-141.

4. Elia M, Normand C, Norman K, Laviano A. A systematic review of the cost and cost effectiveness of using standard oral nutritional supplements in the hospital setting. Clin Nutr. 2016;35(2):370-380. 